\title{
Particle trapping in merging flow junctions by fluid-solute-colloid-boundary interactions
}

\author{
Sangwoo Shin $\odot,{ }^{1, *}$ Jesse T. Ault, ${ }^{2}$ Kazumi Toda-Peters, ${ }^{3}$ and Amy Q. Shen $\circledast^{3}$ \\ ${ }^{1}$ Department of Mechanical Engineering, University of Hawaii at Manoa, Honolulu, Hawaii 96822, USA \\ ${ }^{2}$ School of Engineering, Brown University, Providence, Rhode Island 02912, USA \\ ${ }^{3}$ Okinawa Institute of Science and Technology Graduate University, Onna, Okinawa 904-0495, Japan
}

(Received 23 May 2019; accepted 23 January 2020; published 27 February 2020; corrected 17 April 2020)

\begin{abstract}
Merging of different streams in channel junctions represents a common mixing process that occurs in systems ranging from soda fountains and bathtub faucets to chemical plants and microfluidic devices. Here, we report a spontaneous trapping of colloidal particles in a merging flow junction when the merging streams have a salinity contrast. We show that the particle trapping is a consequence of nonequilibrium interactions between the particles, solutes, channel, and the freestream flow. A delicate balance of transport processes results in a stable near-wall vortex that traps the particles. We use three-dimensional particle visualization and numerical simulations to provide a rigorous understanding of the observed phenomenon. Such a trapping mechanism is unique from the well-known inertial trapping enabled by vortex breakdown [Proc. Natl. Acad. Sci. USA 111, 4770 (2014)], or the solute-mediated trapping enabled by diffusiophoresis [Phys. Rev. X 7, 041038 (2017)], as the current trapping is facilitated by both the solute and the inertial effects, suggesting a new mechanism for particle trapping in flow networks.
\end{abstract}

DOI: 10.1103/PhysRevFluids.5.024304

\section{INTRODUCTION}

Particle-laden flows in channel junctions occur in many applications across a wide range of length scales, from blood flows in capillary networks [1], microfluidic mixing and droplet generation $[2,3]$, and porous media flow to sewer network and groundwater flow [4,5]. While small suspended particles tend to passively follow fluid streamlines in laminar, modest Reynolds number flows, coupled nonlinear effects can result in unexpected particle dynamics in certain systems. For example, a spontaneous particle trapping mechanism was recently identified in branching flows through T- or angle-shaped junctions due to the coupling between inertial particle dynamics and the nonlinear vortex breakdown phenomena [6-11].

The coupling of particle dynamics to other additional physics such as externally imposed temperature or chemical concentration gradients can also cause the particles to deviate from the fluid streamlines and results in interesting particle dynamics [12,13]. Recent examples have shown that for merging flows in channel junctions [14,15] and flow networks [14], suspended particles can also be trapped near the junction when the merging streams contain different solutes. Solute gradients formed at the junction allow the particles to migrate against the flow via diffusiophoresis, leading

\footnotetext{
*sangwoos@hawaii.edu
}

Published by the American Physical Society under the terms of the Creative Commons Attribution 4.0 International license. Further distribution of this work must maintain attribution to the author(s) and the published article's title, journal citation, and DOI. 
to trapping. While this solute-mediated particle trapping contributes to the rapid clogging of porous media [14], it is also proven to be useful for on-chip applications such as DNA preconcentration [14,15], bioseparation [16], and surface characterization [17].

In this paper, we identify a new type of solute-mediated particle trapping in merging T-junctions. We show experimentally and numerically that the nonequilibrium interactions between the particles, solutes, channel wall, and the inlet fluid flow create a spiral sink vortex near the junction that leads to stable particle trapping. We observe a variety of particle trapping morphologies determined by the relative strengths of the nonequilibrium interactions. Three-dimensional (3D) particle visualization and numerical simulations are utilized to visualize the vortex so as to provide a rigorous understanding of the particle trapping phenomenon. The reported trapping mechanism is unique from the inertial trapping enabled by vortex breakdown [6-11], or the solute-mediated trapping enabled by diffusiophoresis [14,15], as the current trapping is facilitated by both the solute and the inertial effects, suggesting a new mechanism for particle trapping in flow networks.

\section{METHODS}

\section{A. Three-dimensional printed glass microfluidic channel}

The 3D-printed glass microfluidic channels were fabricated from a 5-mm-thick polished fused silica substrate (Optostar Ltd., Japan). To fabricate the glass microfluidic channels, we utilized a relatively new subtractive 3D printing fabrication technique known as selective laser-induced etching (SLE) $[18,19]$. SLE is a two-step process that involves the laser irradiation of specific regions within a polished glass substrate and then the removal of those regions by a chemical etching process. We performed the precise volumetric laser irradiation with the commercially available LightFab 3D printer (LightFab, GmbH), which "prints" a modified volume by laser scanning within a fused silica substrate according to an inputted sliced computer-aided design model. Once the laser scanning is complete the modified substrate is submerged in an ultrasonic bath of potassium hydroxide $(\mathrm{KOH})$ where the modified regions are selectively etched at a rate thousand times faster than the unmodified regions of glass.

\section{B. Flow experiments}

Fluorescent colloidal particles (carboxylated-functionalized polystyrene latex, diameter $=1 \mu \mathrm{m}$, ThermoFisher Scientific) were suspended in $\mathrm{NaCl}$ solutions with the initial volume fraction of $0.0001 \%$. The particle $\zeta$ potential was measured by electrophoretic light scattering (Zetasizer Nano-ZS, Malvern Panalytical). The $\mathrm{pH}$ was adjusted by adding $\mathrm{HCl}$ to the $\mathrm{NaCl}$ solution and was measured by a pH meter (HI9813-6, Hanna Instruments). The inlet streams were injected into the microfluidic channel by a syringe pump (Pump 11 Pico Plus Elite, Harvard Apparatus). The particles were visualized with an inverted fluorescence microscope (DMi8, Leica). The time-lapse images were created using ImageJ.

\section{Numerical simulations}

The numerical simulations were performed using finite-volume solvers adapted from the simpleFoam and scalarTransportFoam solvers of the OpenFOAM open-source computational fluid dynamics library [20]. First, we solve for the steady-state fluid and solute concentration profiles using a modified version of the SIMPLE algorithm [21] that uses an iterative relaxation approach to simultaneously solve the Navier-Stokes and continuity equations coupled to an advection-diffusion equation for the solute concentration. Note that this numerical solution involves two-way coupling since the fluid velocity sets the advection term for the solute concentration, and the solute concentration gradient sets the approximate wall slip boundary condition on the fluid velocity through the action of diffusioosmosis. Once this solver has relaxed to a steady-state solution, we integrate an unsteady advection-diffusion equation for the particle concentration $n$ with a modified advection term given by $\boldsymbol{u}+\mathcal{M}_{\mathrm{p}} \boldsymbol{\nabla} \ln c$ to include the influence of diffusiophoresis on the particle 

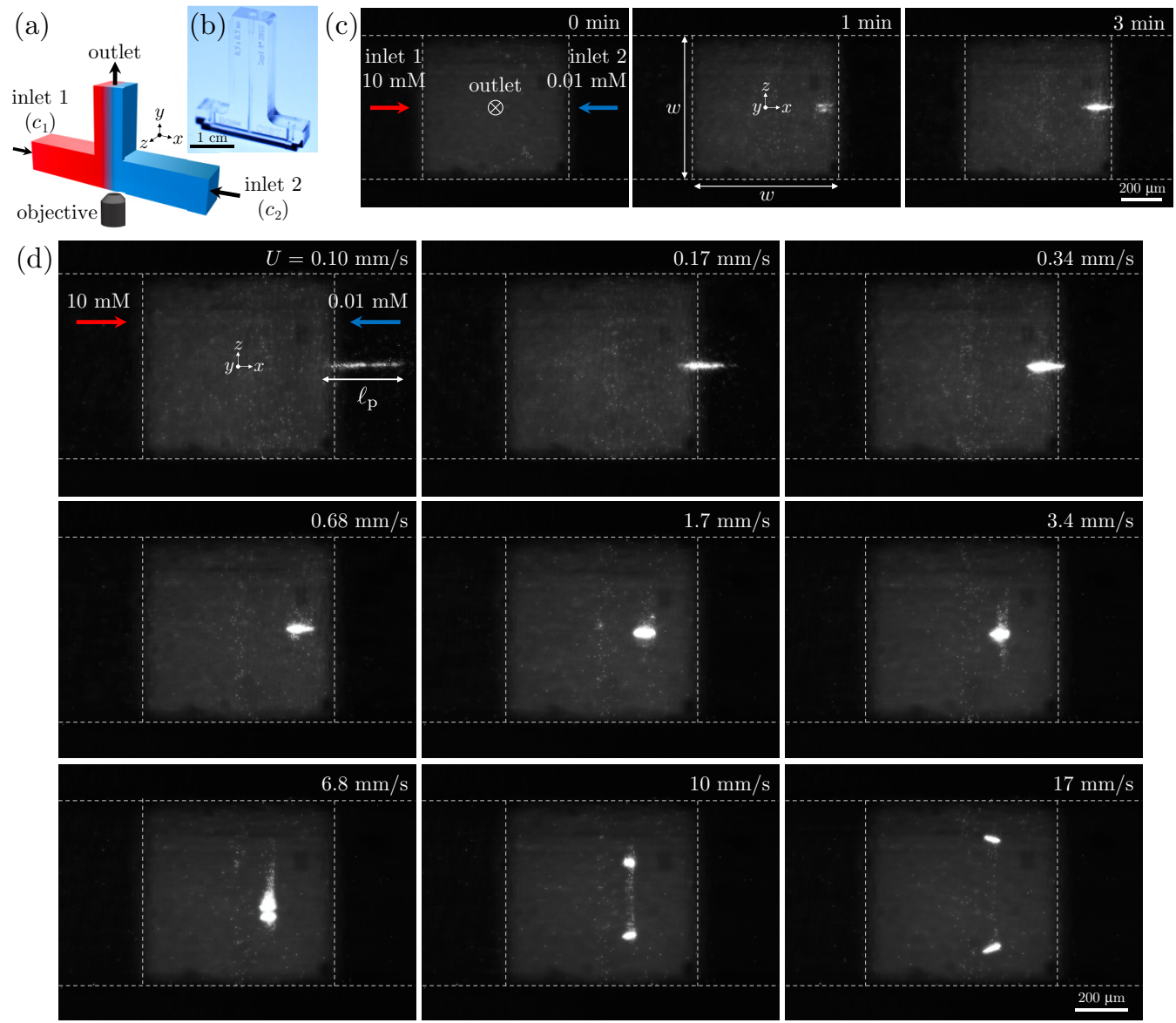

FIG. 1. Particle trapping induced by merging of different saline solutions at a T-junction. (a) Schematic of a symmetric T-junction with merging flows of solute concentrations $c_{1}$ and $c_{2}$. (b) A photograph of a glass microfluidic T-junction device made by selective laser-induced etching. (c) Image sequence of fluorescent colloidal particles (carboxylate-modified polystyrene latex, $1 \mu \mathrm{m}$ in diameter) accumulating near the junction by merging of two colloidal suspensions with different solute concentrations $\left(\mathrm{NaCl}, c_{1}=10 \mathrm{mM}\right.$, $\left.c_{2}=0.01 \mathrm{mM}\right)$. Inlet mean flow speed is $U=0.34 \mathrm{~mm} / \mathrm{s}$. The channel has a square cross section with width $w=0.7 \mathrm{~mm}$ throughout the entire channel. See also Movie 1 in the Supplemental Material [22]. (d) Particle trapping versus the inlet mean flow speed. The images are taken at $5 \mathrm{~min}$ after the flow has started.

dynamics. Here, we assume an initial particle distribution of $n(\boldsymbol{r}, t)=1$ and integrate forward in time to observe the locations at which particles accumulate in the system. The magnitude of $n$ at a fixed time represents the degree to which particles are accumulated at a specific location.

\section{RESULTS AND DISCUSSION}

\section{A. Particle trapping in merging $\mathrm{T}$-junction}

A schematic of a merging T-junction is presented in Fig. 1(a). Two streams with the same flow rates having different $\mathrm{NaCl}$ concentrations are injected through the side inlets; the more saline solution flows from the left (inlet 1 , concentration $c_{1}=10 \mathrm{mM}$ ) and the less saline solution flows from the right (inlet 2, $c_{2}=0.01 \mathrm{mM}$ ). We set the Cartesian origin to be at the stagnation point of an otherwise uniform merging flow in a symmetric T-junction. 
For the experiments, we utilize a microfluidic T-junction device fabricated by selective laserinduced etching [18,19], as shown in Fig. 1(b). The channel has a square cross-section with width $w=0.7 \mathrm{~mm}$ throughout the entire channel. This device allows flow visualization from various perspectives, including from underneath the junction $(x-z$ plane $)$, which is difficult to achieve with devices made by soft lithography.

When particles are added to the streams, we observe a sudden trapping of colloidal particles near the junction. Fluorescence microscope images of fluorescently labeled colloidal particles are shown in Fig. 1(c) (see also Movie 1 in the Supplemental Material [22]). The images are taken from underneath the junction ( $x-z$ plane). The particles are carboxylate-functionalized polystyrene latex spheres with 1 -m diameters. The inlet mean flow speed in the figure is $U=0.34 \mathrm{~mm} / \mathrm{s}$. The image sequence shows that the trapped particles continue to accumulate at a localized region over time, forming a stable particle cluster.

The morphology of the particle cluster is observed to depend on the inlet flow speed. Figure 1(d) shows the trapped particles at various inlet mean flow speeds ranging from $U=0.10$ to $17 \mathrm{~mm} / \mathrm{s}$. These flow speeds correspond to the Reynolds numbers of $\operatorname{Re}=U w / v=0.040-6.6$ and the solute Péclet numbers of $\mathrm{Pe}=U w / D_{\mathrm{s}}=22-3700$, where $v$ and $D_{\mathrm{s}}$ are, respectively, the momentum and solute diffusivities. The images are taken at 5 minutes after the flow has started. At low inlet flow speed $(U<0.5 \mathrm{~mm} / \mathrm{s} ; \operatorname{Re}<0.19)$, the particles form a long, narrow band that aligns along the centerline ( $x$ axis) of the inlet channel. However, as the flow rate is increased, the particle cluster becomes compact and tends to form more toward the origin. When $U>5 \mathrm{~mm} / \mathrm{s}(\operatorname{Re}>1.9)$, the trapped particles split into two distinct particle clusters that are symmetric about the $x$ axis. We note that in the absence of solute contrast between the two inlet streams, the particles do not experience any trapping, but leave the junction with the fluid flow.

\section{B. Solute-gradient-induced particle vortex}

Because of the solute gradient developed at the interface of the two inlet streams, two possible nonequilibrium transport mechanisms may emerge that can impact the particle transport, namely, diffusioosmosis and diffusiophoresis [23-26]. In the presence of solute gradients, near a solid-liquid interface the solute gradients can induce fluid flow relative to the solid surface to relax the interfacial free energy imbalance; the solute gradients can develop an osmotic pressure gradient near the interface, thus causing osmotic flow (chemiosmosis). The solute gradients can also drive electroosmosis due to the diffusion potential gradient developed by the diffusion of various ionic species. The combined process is referred to as diffusioosmosis, where the local fluid dynamics can be approximated as a wall slip flow given by $\boldsymbol{u}_{\mathrm{w}}=-\mathcal{M}_{\mathrm{w}} \nabla \ln c$, where $\mathcal{M}_{\mathrm{w}}$ is the (wall) diffusioosmotic mobility, which is the sum of chemiosmotic and electroosmotic mobilities. If the solid is freely suspended (e.g., colloidal particles), then this wall slip flow can result in particle motion in the opposite direction, a process known as diffusiophoresis. The particle diffusiophoretic velocity is given as $\boldsymbol{u}_{\mathrm{p}}=\mathcal{M}_{\mathrm{p}} \boldsymbol{\nabla} \ln c$, where $\mathcal{M}_{\mathrm{p}}$ is the (particle) diffusiophoretic mobility. $\mathcal{M}_{\mathrm{w}}$ and $\mathcal{M}_{\mathrm{p}}$ represent the efficacy of, respectively, diffusioosmosis and diffusiophoresis for a given solid-liquid system.

The surface charge of both the channel (fused silica) and the particles (carboxylate-functionalized polystyrene) gives rise to the electrolytic diffusioosmosis/-phoresis [27], which is generally stronger than the nonelectrolytic counterpart. We find that the observed particle trapping phenomenon is a consequence of complex interactions between diffusioosmosis, diffusiophoresis, and inlet fluid flow. Near the liquid-liquid interface, a solute gradient is established that drives diffusioosmosis toward the less saline stream (positive $x$ direction) due to the positive $\mathcal{M}_{\mathrm{w}}$ in the current system. Since diffusioosmosis is effectively a slip flow at the macroscale, the combination of diffusioosmosis and the inlet fluid flow results in a vortical flow near the channel wall $[28,29]$.

This gradient-induced fluid vortex is presented in Fig. 2. A 2D numerical simulation of the steady-state solute distribution $c$ in a symmetric T-junction is shown in Fig. 2(a). The simulation incorporates diffusioosmosis as a slip boundary condition on the channel wall, which is given as 

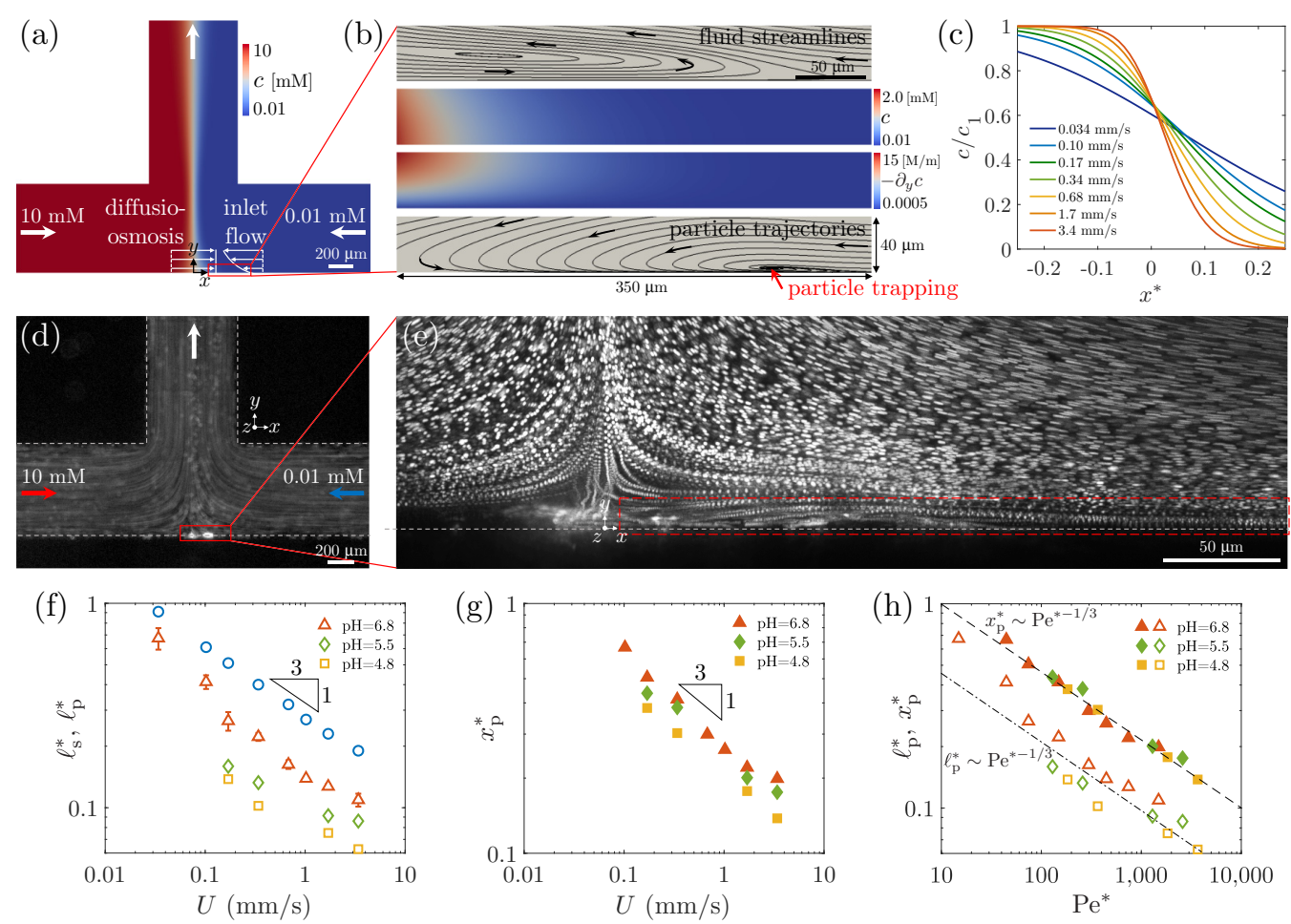

FIG. 2. Particle trapping is a consequence of interactions between diffusiophoresis, diffusioosmosis, and inlet fluid flow. (a) Solute distribution $c$ in a 2D T-junction. Near the interface between two streams, a solute gradient is established that induces diffusioosmosis directed toward the less saline stream (positive $x$ direction). Because diffusioosmosis is a slip flow, the combination of diffusioosmosis and inlet fluid flow results in a vortical flow near the wall. (b) Close-up simulation results near the wall [red box in (a)] showing fluid streamlines, $c,-\partial_{y} c$, and particle trajectories. (c) Solute concentration adjacent to the wall $(y \rightarrow 0)$ normalized by the bulk solute concentration of more saline stream $\left(c_{1}\right)$ near the interface of the two solutions for various inlet mean flow speeds. $x$ is rescaled by the channel width $w, x^{*}=x / w$. (d) A snapshot of the T-junction observed from the front ( $x-y$ plane). A small region of the trapped particles is denoted by a red box. (e) A reconstructed image of the particles in the red box in (c) by overlaying image sequence to visualize the particle trajectories. (f) Normalized solute dispersion length $\ell_{\mathrm{s}}^{*}=\ell_{\mathrm{s}} / w$, normalized particle cluster length $\ell_{\mathrm{p}}^{*}=\ell_{\mathrm{p}} / w$, and $(\mathrm{g})$ normalized particle cluster location $x_{\mathrm{p}}^{*}=x_{\mathrm{p}} / w$ versus the inlet mean flow speed $U . \ell_{\mathrm{s}}^{*}$ and $\ell_{\mathrm{p}}^{*}$ for various wall diffusioosmotic conditions are presented by altering the solution $\mathrm{pH}$. Open blue circle represents $\ell_{\mathrm{s}}^{*}$, open triangle, diamond, and square represent $\ell_{\mathrm{p}}^{*}$ at various $\mathrm{pH}$ conditions, and closed triangle, diamond, and square represent $x_{\mathrm{p}}^{*}$ at various $\mathrm{pH}$ conditions. (h) $\ell_{\mathrm{s}}^{*}$ and $\ell_{\mathrm{p}}^{*}$ replotted with $\mathrm{Pe}^{*}=U w / \mathcal{M}_{\mathrm{w}}$.

$\boldsymbol{u}_{\mathrm{w}}=-\mathcal{M}_{\mathrm{w}} \nabla_{s} \ln c$, where $\nabla_{s}$ is the surface gradient operator. We assume $\mathcal{M}_{\mathrm{w}}=1600 \mu \mathrm{m}^{2} / \mathrm{s}$ unless otherwise noted, which corresponds to a value for a negatively-charged surface in contact with aqueous $\mathrm{NaCl}$ solution. The wall $\zeta$ potential is $\approx-110 \mathrm{mV}$, which is a typical value for fused silica in $1 \mathrm{mM} \mathrm{NaCl}$ or $\mathrm{KCl}$ near neutral $\mathrm{pH}$ conditions [30-34]. We also estimate $\mathcal{M}_{\mathrm{p}}=830 \mu \mathrm{m}^{2} / \mathrm{s}$ from the measured particle $\zeta$ potential $\approx-75 \mathrm{mV}$ in $5 \mathrm{mM} \mathrm{NaCl}$ [35].

Figure 2(b) is a close-up of the simulation results near the wall where the vortex is present [red box in Fig. 2(a)]. The computed fluid streamlines identify the gradient-induced vortex [topmost panel of Fig. 2(b)]. The size of the vortex is essentially determined by the solute dispersion length $\ell_{\mathrm{s}}$, which decreases as the flow speed is increased [Fig. 2(c)]. The size $\ell_{\mathrm{p}}$ and the location $x_{\mathrm{p}}$ of the particle cluster are also set by $\ell_{\mathrm{s}}$, as will be discussed later more in detail. 
In short, the combination of the solute-gradient-induced diffusioosmosis and the free-stream flow velocity away from the channel walls result in the formation of a fluid vortex near the channel bottom. However, the formation of this vortex is not sufficient to drive particle accumulation in the absence of other effects since the velocity field for a cloud of passive tracer particles in an incompressible flow must also be incompressible. Therefore, an additional factor, specifically diffusiophoresis, is required to drive the particles to accumulate at a kinematically stable fixed point.

Since the solutes closer to the wall $(y \rightarrow 0)$ are dispersed more broadly in the $x$ direction due to the lesser influence of the advection [second panel of Fig. 2(b)], the solutes have concentration gradients not only in the $x$ but also in the $y$ direction [third panel of Fig. 2(b)]. This gradient $\partial_{y} c$ causes the particles to actively migrate toward the wall by diffusiophoresis due to the positive $\mathcal{M}_{\mathrm{p}}$, producing a particle vortex that is distinct from the fluid vortex. This particle vortex acts as a stable fixed point for the particles, and their trajectories spiral toward the core of this vortex. Compared to the fluid vortex, the particle vortex is shifted more toward the negative $y$ direction, thus forming very near to the channel bottom [bottommost panel of Fig. 2(b)]. The particles ultimately accumulate at the vortex core, as denoted by the red arrow. Moreover, the particle vortex is located more toward the upstream (positive $x$ direction) due to the lesser influence of the inlet fluid flow close to the channel bottom.

The colloidal vortices can be identified in the experiments by observing the particles from the front ( $x-y$ plane). Figure 2(d) shows a snapshot of the T-junction in the $x-y$ plane where the particle cluster can be identified inside the red box. To visualize the particle trajectories, we construct a time-lapse image of the particles from the red box in Fig. 2(d) by overlaying the image sequence, which is shown in Fig. 2(e). The particle vortex can be identified inside the red dashed box, which is formed within several microns from the wall.

We note that the length $\ell_{\mathrm{p}}$ and the location $x_{\mathrm{p}}$ of the particle cluster, and the solute dispersion length $\ell_{\mathrm{s}}$ all decrease with the inlet flow speed approximately to the power of $-1 / 3$ as shown in Figs. 2(f) and $2(\mathrm{~g}) . \ell_{\mathrm{s}}$ is determined from the simulations in Fig. 2(c), whereas $\ell_{\mathrm{p}}$ and $x_{\mathrm{p}}$ are measured experimentally for various wall diffusioosmotic conditions by altering the solution $\mathrm{pH}$; this will be discussed later in Sec. III D in more detail. $x_{\mathrm{p}}$ indicates the distance of the center of the cluster away from the origin. Here, we seek to explain these relationships with a simple scaling argument. For the case of 2D symmetric merging T-junction flow with no-slip boundaries, the fluid velocity $\boldsymbol{v}=\left(u_{x}, u_{y}\right)$ near the stagnation point $(x, y)=(0,0)$ behaves like $u_{x} \sim-U x y$ and $u_{y} \sim U y^{2}$. Then, balancing the advection and diffusion terms in a $2 \mathrm{D}$ advection-diffusion equation for the solute, we find that $\ell_{\mathrm{s}} \sim\left(D_{\mathrm{s}} / U\right)^{1 / 3} \sim U^{-1 / 3}$. Finally, the characteristic length scale for solute dispersion effectively sets the characteristic length scale for particle accumulation, and so we also expect $\left\{\ell_{\mathrm{p}}, x_{\mathrm{p}}\right\} \sim \ell_{\mathrm{s}}$. This could alternately be seen by extending the scaling analysis to a modified advection-diffusion equation for the particle concentration that includes the contribution from diffusioosmosis. Since both $\ell_{\mathrm{p}}$ and $x_{\mathrm{p}}$ are determined by the balance between the inlet fluid flow and diffusioosmosis, the ratio of the two transport processes gives a Péclet-like dimensionless number $\mathrm{Pe}^{*}=U w / \mathcal{M}_{\mathrm{w}}$ that sets the particle cluster size and location. Replotting $\ell_{\mathrm{p}}^{*}\left(=\ell_{\mathrm{p}} / w\right)$ and $x_{\mathrm{p}}^{*}\left(=x_{\mathrm{p}} / w\right)$ with $\mathrm{Pe}^{*}$ for various $\mathcal{M}_{\mathrm{w}}$ conditions show a good overlap as shown in Fig. 2(h).

A full 3D numerical simulation further reveals the details of the particle trapping phenomenon. Figure 3(a) shows close-up images of the computed particle vortex at $U=0.34 \mathrm{~mm} / \mathrm{s}$. These are elongated by a factor of 10 in the $y$ direction for better visualization since the vortex occurs only within several micrometers from the wall. The 3D simulation not only shows the particle entrainment occurring in the $x-y$ plane, which is also predicted in the $2 \mathrm{D}$ simulations, but also reveals that the particles converge from the sides toward the center $(z \rightarrow 0)$ due to the solute gradients established in the $z$ direction; this will be discussed later in Sec. III C in detail. The particle entrainment is also observed experimentally in Fig. 3(b), which is a cropped image from the dashed red box in Fig. 2(e), but also elongated 10 times in the $y$ direction for comparison. The trajectories show a good match with the simulations where the particles gradually migrate away from the wall 
(a)

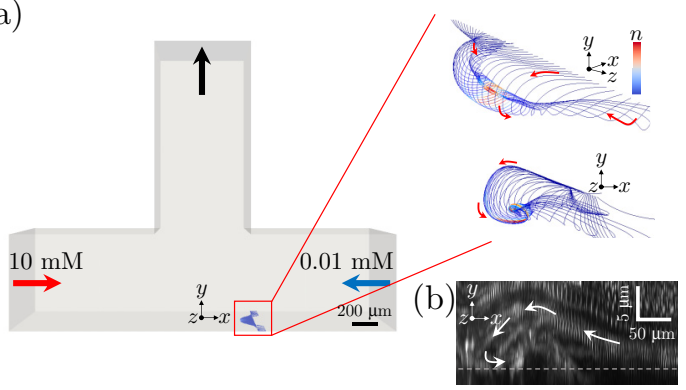

(d) $10 \mathrm{mM}$
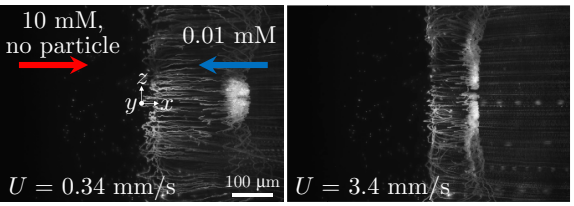

(e)

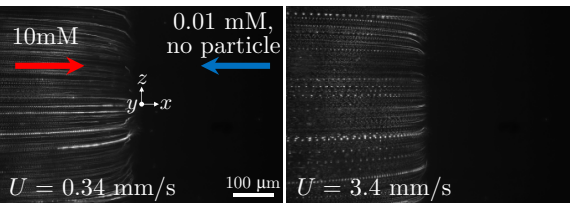

(c)
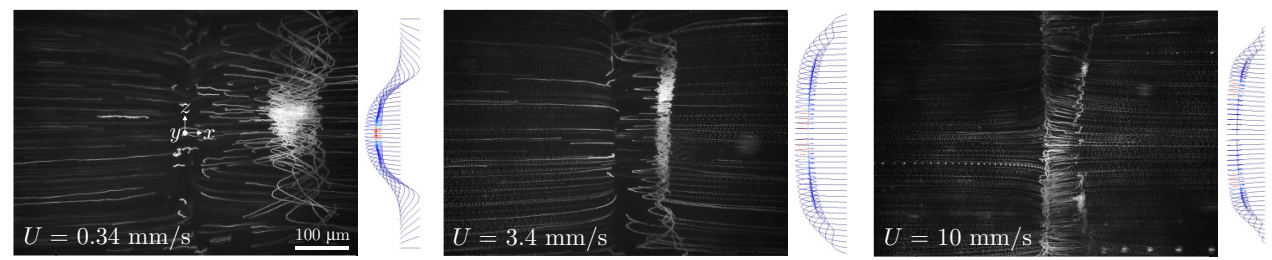

FIG. 3. Colloidal vortices. (a) A full 3D simulation of the colloidal vortex at $U=0.34 \mathrm{~mm} / \mathrm{s}$. Close-up images of the vortex from the red box are elongated by a factor of 10 times in the $y$ direction for a better visualization. (b) Particle trajectories cropped from the red box in Fig. 2(d). The image is also elongated 10 times in the $y$ direction for comparison. (c) Particle trajectories in $x-z$ planes for various flow rates. Simulated particle trajectories are also presented for comparison. See also Movie 2 in the Supplemental Material [22]. (d), (e) Particle trajectories for the merging flows having colloidal particles only in either (d) the less saline $(0.01 \mathrm{mM})$ or (e) the more saline $(10 \mathrm{mM})$ solution. Only the particles coming from the less saline side are trapped. See also Movie 3 in the Supplemental Material [22].

as they move downstream toward the junction centerplane and then are rapidly entrained into the vortex, creating airfoil-like particle trajectories

The colloidal vortices are also identified by observing from underneath, i.e., from the $x-z$ plane. Figure 3(c) shows the time-lapse images of the particle trajectories near the interface in the $x-z$ plane at various inlet flow speeds $(U=0.34-10 \mathrm{~m} / \mathrm{s}$; see also Movie 2 in the Supplemental Material [22]). Computed 3D colloidal vortices are laid side-by-side for comparison. The spiral trajectories converging toward the center clearly show the lateral particle entrainment, as predicted by the 3D simulation.

The particle trajectories also suggest that the particles suspended in the more saline side do not experience trapping but instead leave the junction without being entrained into the vortex. We confirm this observation by performing experiments with particles suspended only in one stream while excluding the other. Figures 3(d) and 3(e) show the time-lapse images when the particles are suspended only in either the less saline [Fig. 3(d)] or the more saline [Fig. 3(e)] solution; see also Movie 3 in the Supplemental Material [22]. Regardless of the inlet flow speed, only the particles coming from the less saline side are trapped. This is due to the fact that the solute gradient in the $y$ direction $\partial_{y} c$ is positive in the more saline side near the interface, as opposed to the less saline side, where $\partial_{y} c$ is negative. This gradient causes the particles in the more saline side to move away from the wall by diffusiophoresis so that the particles are easily advected by the inlet fluid flow and leave the junction.

We note that the gravity plays a negligible role in the particle trapping. For instance, the typical speed of diffusioosmosis in this study is estimated as $u_{\mathrm{w}} \approx \frac{\mathcal{M}_{\mathrm{w}}}{w} \sim \mathcal{O}(1) \mu \mathrm{m} / \mathrm{s}$, whereas the particle sedimentation speed is $u_{\mathrm{s}}=\frac{\Delta \rho D^{2} g}{18 \mu}=2 \mathrm{~nm} / \mathrm{s}$. Here, $\Delta \rho=0.04 \mathrm{~g} / \mathrm{cm}^{3}$ is the density difference, $D=1 \mu \mathrm{m}$ is the particle diameter, $\mu=1 \mathrm{mPas}$ is the solution viscosity, and $g$ is the gravitational acceleration. Since the sedimentation occurs three orders of magnitude slower than 
(a)
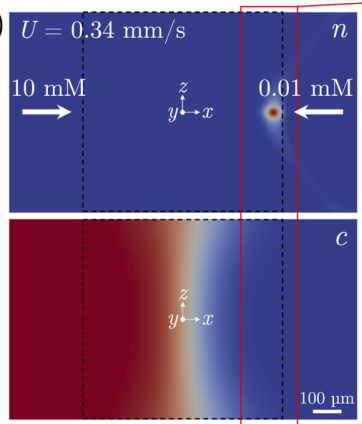

$100 \mu \mathrm{m}$
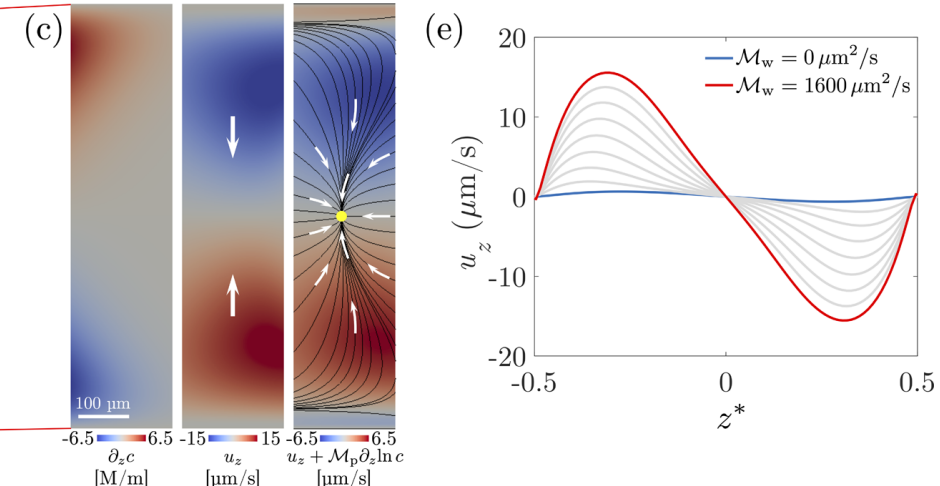

(b)

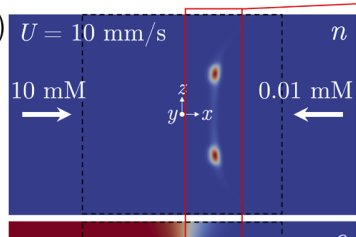

(d)
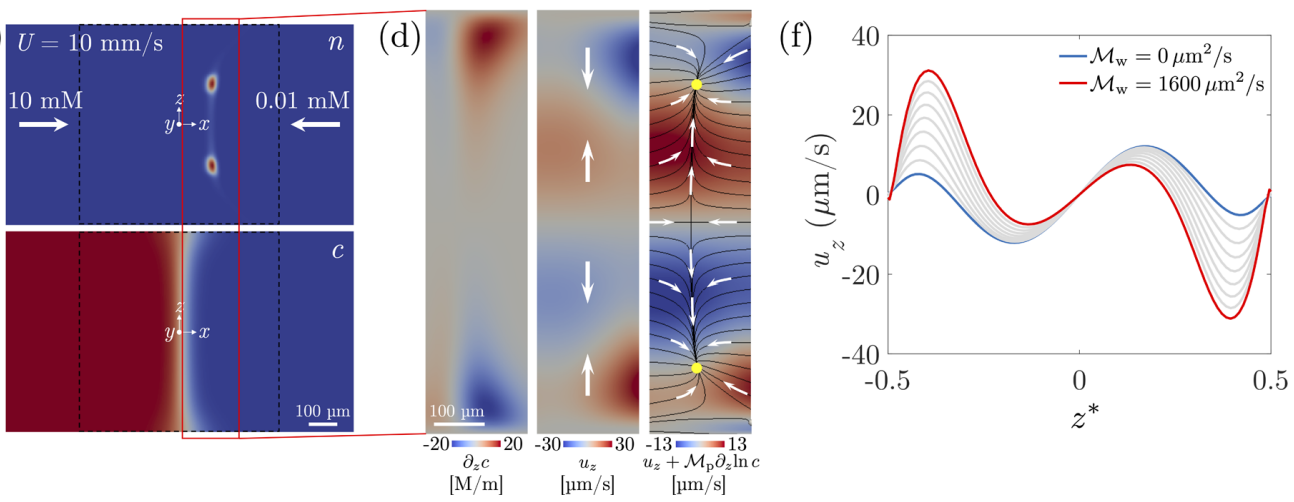

FIG. 4. Solute transport determines the morphology of the particle cluster. (a), (b) 3D simulations of the particle $n$ and the solute $c$ distributions at (a) $U=0.34 \mathrm{~mm} / \mathrm{s}$ and (b) $10 \mathrm{~mm} / \mathrm{s}$ adjacent to the channel wall. (c), (d) Close-up images of the solute gradient in the $z$ direction $\left(\partial_{z} c\right)$, flow velocity in the $z$ direction $\left(u_{z}\right)$, and the particle velocity in the $z$ direction $\left(u_{z}+\mathcal{M}_{\mathrm{p}} \partial_{z} \ln c\right)$ from the red boxes in $(\mathrm{a}, \mathrm{b})$. Particle trajectories are overlaid on the particle velocity panels. Yellow dots represent the particle stagnation points. (e), (f) $u_{z}$ taken along the $z$ axis that intersects the maximum particle concentration regions $\left[x^{*}=0.45\right.$ in (e); $x^{*}=0.16$ in (f)]. Blue curves indicate $u_{z}$ in the absence of diffusioosmosis $\left(\mathcal{M}_{\mathrm{w}}=0 \mu \mathrm{m}^{2} / \mathrm{s}\right)$. Red curves indicate $u_{z}$ in the presence of diffusioosmosis $\left(\mathcal{M}_{\mathrm{w}}=1600 \mu \mathrm{m}^{2} / \mathrm{s}\right)$. Gray curves indicate $u_{z}$ in between the two diffusioosmosis conditions with an interval of $200 \mu \mathrm{m}^{2} / \mathrm{s}$. $z$ is rescaled by the channel width $w, z^{*}=z / w$. All the data are taken at $y=5 \mu \mathrm{m}$.

the diffusioosmosis, we can safely neglect gravity in our system. However, the gravity may become significant for inertial particles, and either promote or suppress the particle trapping depending on the channel orientation, which may be important in practical applications.

\section{Formation of double particle clusters}

We now turn our attention to the formation of double clusters at high inlet flow speeds, as shown earlier in Fig. 1(d) and also in Fig. 3(c) at $U=10 \mathrm{~mm} / \mathrm{s}$. While the particle cluster length $\ell_{\mathrm{p}}$ decreases with increasing flow speed, which can be described by $2 \mathrm{D}$ arguments, further increasing the flow speed leads to splitting of the particle cluster in the direction transverse to the inlet flow, which is clearly a 3D effect. A full 3D numerical simulation also reveals similar behavior in which the particle cluster transitions from a single [top panel of Fig. 4(a)] to a double cluster [top panel of Fig. 4(b)] as the inlet flow speed is increased.

Because the channel is finite in the $z$ direction in 3D, the solutes are more broadly distributed at the interface closer to the wall also in the $z$ direction $\left[z^{*}=z / w \rightarrow \pm 1 / 2\right.$; bottom panels of Figs. 4(a) 

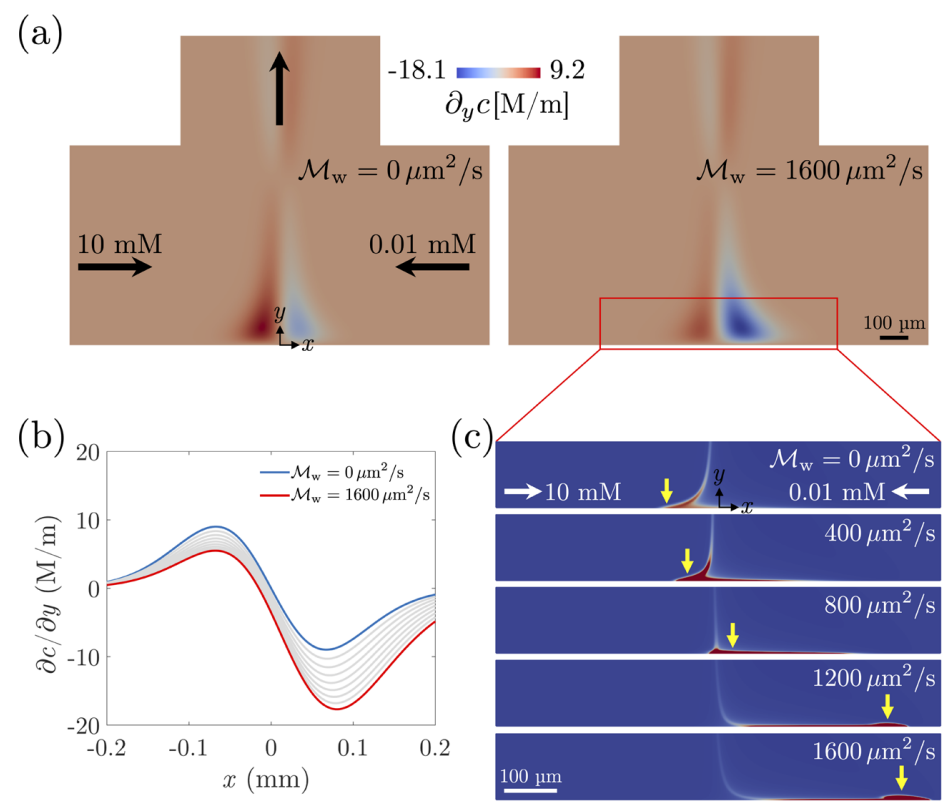

(d)

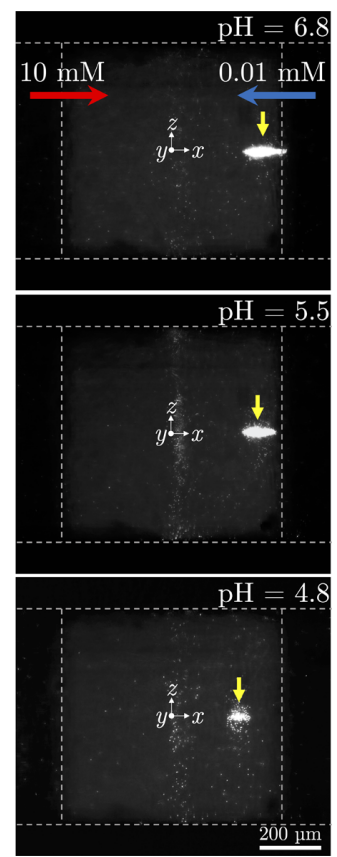

FIG. 5. Diffusioosmosis enhances diffusiophoresis. (a), (b) 2D simulations of (a) the solute gradient in the $y$ direction $\partial_{y} c$ and (b) their distribution near the interface at $y=50 \mu \mathrm{m}$. The gradient is plotted with an interval of $200 \mu \mathrm{m}^{2} / \mathrm{s}$. (c) Close-up images of the particle concentration $n$ from the red box in (a) for various wall diffusioosmotic mobilities $\mathcal{M}_{\mathrm{w}}$. (d) Particle distributions in $x-z$ plane for various $\mathrm{pH}$ values. The images are taken at $5 \mathrm{~min}$ after the flow has started. Yellow arrows in (c), (d) indicate the locations of the center of the particle cluster $x_{\mathrm{p}} . U=0.34 \mathrm{~mm} / \mathrm{s}$ for all cases.

and 4(b)]. This creates an additional solute gradient in the $z$ direction $\partial_{z} c$ [left panels of Figs. 4(c) and 4(d)], which drives diffusioosmosis that converges toward the center $\left(z^{*} \rightarrow 0\right)$. While this diffusioosmotic flow occurs regardless of the inlet flow speed, the converging diffusioosmotic flow extends over the entire channel width when the inlet flow speed is relatively low $(U=0.34 \mathrm{~mm} / \mathrm{s}$; $\operatorname{Re}=0.13)$ due to the solutes being more dispersed. In contrast, when the flow speed is high $(U=10 \mathrm{~mm} / \mathrm{s} ; \operatorname{Re}=4.0)$, the stronger advection makes the solutes less dispersed, thus confining $\partial_{z} c$ only close to the wall [left panels of Figs. 4(c) and 4(d)]. Consequently, the converging diffusioosmosis only extends from the wall to a certain extent whereas the middle region of the channel is dominated by the inlet fluid flow, which diverges from the center toward the wall.

This behavior can be identified from the contribution of diffusioosmosis on the flow velocity, as shown in Figs. 4(e) and 4(f) where the red and blue curves represent, respectively, the $z$ velocity $u_{z}$ in the presence and the absence of diffusioosmosis. At $U=0.34 \mathrm{~mm} / \mathrm{s}$ the diffusioosmosis has a strong impact in the $z$-velocity $u_{z}$ across the whole channel width [Fig. 4(e)], whereas the diffusioosmosis influences $u_{z}$ mostly near the wall at $U=10 \mathrm{~mm} / \mathrm{s}$ [Fig. 4(f)]. The diverging fluid flow is a consequence of the early onset of Dean-like vortices near the junction having high curvature [36], which is clearly an inertial effect. The combination of diverging flow from the center and the converging diffusioosmosis from the wall makes a pair of converging flows that are symmetric about the $x$ axis, leading to the formation of the double particle cluster at high inlet flow speed. The $z$ velocity $u_{z}$ presented in the center panels of Figs. 4(c) and 4(d) and the plots in Figs. 4(e) and 4(f) reveal the double converging flows at $U=10 \mathrm{~mm} / \mathrm{s}$ [Figs. 4(d) and 4(f)], whereas a single converging flow that is only dominated by diffusioosmosis is observed at $U=0.34 \mathrm{~mm} / \mathrm{s}$ [Figs. 4 (c) and 4(e)]. 
(a)
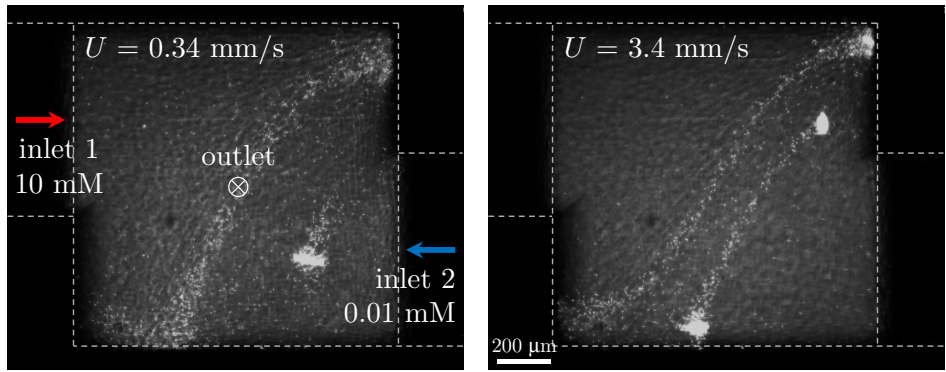

(b)
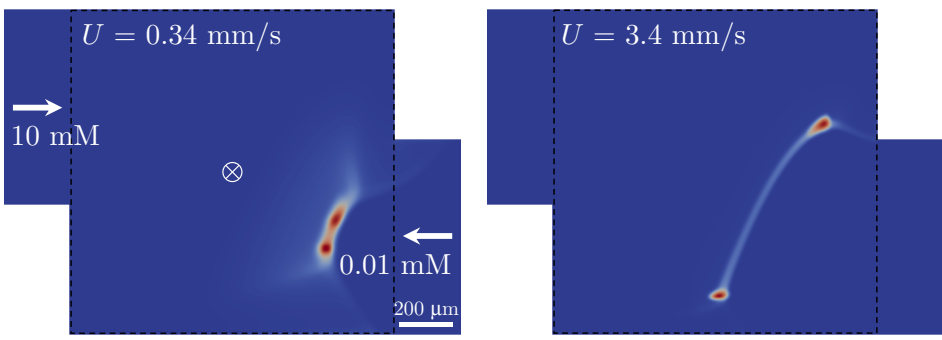

FIG. 6. Particle trapping also occurs in other merging flow geometries. Particle trapping in a staggered T-junction where two inlets are offset. The channel has a square cross section, but with different widths for the inlets and the outlet; $0.7 \mathrm{~mm}$ for the inlets and $1.2 \mathrm{~mm}$ for the outlet. (a) Experimental and (b) full 3D numerical simulation results for various inlet mean flow speeds. See also Movie 4 in the Supplemental Material [22].

The particle velocity in the $z$ direction, which is the sum of fluid flow and diffusiophoresis, i.e., $u_{z}+\mathcal{M}_{\mathrm{p}} \partial_{z} \ln c$, also shows similar behavior [right panels of Figs. 4(c) and 4(d)]. The overlaid particle trajectories clearly show that there are two stable particle stagnation points at $U=10 \mathrm{~mm} / \mathrm{s}$, as indicated by yellow dots. This confirms the transition from a single cluster at low flow speed to a double cluster at high flow speed.

\section{Impact of diffusioosmosis on particle trapping}

As we have seen so far, the interaction between diffusioosmosis, diffusiophoresis, and inlet fluid flow enables the particle trapping. We emphasize that the wall diffusioosmosis accelerates the colloid diffusiophoresis by altering the solute distribution, which subsequently leads to enhanced cluster formation. The effect of diffusioosmosis on the particle trapping phenomenon is presented in Fig. 5. In the absence of diffusioosmosis $\left(\left[\mathcal{M}_{\mathrm{w}}=0 \mu \mathrm{m}^{2} / \mathrm{s}\right.\right.$; left panel of Fig. 5(a)], the solute and its gradient are distributed antisymmetrically about the origin in the $x$ direction. In contrast, in the presence of diffusioosmosis $\left(\left[\mathcal{M}_{\mathrm{w}}=1600 \mu \mathrm{m}^{2} / \mathrm{s}\right.\right.$; right panel of Fig. 5(a)], the solutes are transported further to the less saline side (positive $x$ direction) by diffusioosmosis, resulting in an asymmetric distribution of the solutes. Consequently, the vertical solute gradient $\partial_{y} c$ near the less saline side is enhanced [Fig. 5(b)], leading to stronger particle trapping. Also, the particle cluster is formed more upstream as the wall diffusioosmotic mobility is increased, as confirmed in Fig. 5(c); yellow arrows indicate the location of the maximum particle concentration. The results also show that the particle cluster can be formed even at the high saline side when $\mathcal{M}_{\mathrm{w}} \ll \mathcal{M}_{\mathrm{p}}$ due to the diffusiophoresis being the dominant migration mechanism.

The diffusioosmosis-dependent particle dynamics are also verified experimentally by varying the $\mathrm{pH}$ of the solutions [Fig. 5(d)]. For the tested $\mathrm{pH}$ range $(\mathrm{pH}=4.8-6.8)$, the $\zeta$ potential of the wall (fused silica) changes over 50\% [37], whereas the particle (carboxylated polystyrene) $\zeta$ potential varies less than $10 \%$ [38]). This allows us to decouple diffusiophoresis and diffusioosmosis by reducing the wall diffusioosmosis significantly while effectively keeping the particle diffusiophoresis as is. For estimating $\mathcal{M}_{\mathrm{w}}$ values at different $\mathrm{pH}$ values in Fig. 2(h), we assume 
a linear change in the wall $\zeta$ potential with respect to the solution $\mathrm{pH}$, which is a reasonable approximation below $\mathrm{pH}=7$ for glass [30,31,37]. By setting the isoelectric point at $\mathrm{pH}=2$, the wall $\zeta$ potential is estimated as $-80 \mathrm{mV}$ and $-64 \mathrm{mV}$ for $\mathrm{pH}=5.5$ and 4.8 , respectively, which correspond to $\mathcal{M}_{\mathrm{w}}=920$ and $650 \mu \mathrm{m}^{2} / \mathrm{s}$. As the $\mathrm{pH}$ drops from 6.8 to 4.8 , the particle clusters are formed more downstream toward the origin, from $x_{\mathrm{p}}^{*}=0.41$ to 0.30 , as indicated by the yellow arrows in Fig. 5(d). This behavior occurs at various flow speeds, as shown in Figs. 2(g) and 2(h). As expected, this is due to the weakened diffusioosmosis, which agrees with the numerical prediction in Fig. 5(c). The particle cluster also becomes more dispersed in the lateral direction as the laterally converging diffusioosmosis in the $z$ direction becomes weaker, making the particles less compact. Such a diffusioosmosis-dependent cluster morphology suggests a unique way of measuring the wall $\zeta$ potential based on particle visualization, which otherwise requires additional electrical measurements [39-41].

\section{CONCLUSIONS}

Our study demonstrates a good example of how nonlinear, coupled fluid-solute-colloid-boundary dynamics can result in peculiar particle behavior in simple flow systems. With a 3D-printed glass microfluidic device that offers versatile visualization and full 3D numerical simulations, we have identified the formation of stable vortices in a merging T-junction when the inlet streams have a salinity difference. Driven by the interactions between the wall diffusioosmosis, particle diffusiophoresis, and the inlet fluid flow, the vortices enable robust trapping of colloidal particles near the junction. We emphasize that the reported particle trapping is a completely different mechanism from the trapping enabled by the inertial vortex breakdown [6-11] or the solute-mediated trapping enabled by the diffusiophoresis [14,15], as the current trapping is enabled by the combination of both the solute-mediated and the inertial effects. Since the particle trapping is driven by the presence of solute gradients, the trapping can happen in other flow geometries as well. For example, Fig. 6 shows experimentally and numerically that the suspended particles form similar colloidal clusters in a staggered T-junction in which the two inlets are offset (see also Movie 4 in the Supplemental Material [22]). Since asymmetric channel geometries are found in a number of transport systems such as xylem/phloem transport [42], blood flow [43], seepage networks [44], and microfluidic mixers [45], the presented work documents and characterizes a unique particle trapping mechanism potentially found in a wide range of common flow systems. Further, the fact that the particle trapping occurs in low Reynolds number conditions with aid of solutes implies that similar dynamics may arise in biological and physiological environments where a variety of solutes and electrolytes are subjected to a mild fluid flow [46]. Last, due to the extreme near-wall dynamics of the identified trapping mechanism, similar solute-gradient-induced vortices or accumulated particle structures may have been undetected and unappreciated in many microfluidic merging devices [47-49], especially those manufactured with traditional soft lithography without the ability to resolve the near-wall regions.

\section{ACKNOWLEDGMENTS}

We thank Bhargav Rallabandi for helpful discussions. S.S. acknowledges support from the National Science Foundation under Grant No. CBET-1930691. K.T. and A.Q.S. thank Okinawa Institute of Science and Technology Graduate University (OIST) for their financial support, with subsidy funding from the Cabinet Office, Government of Japan. A.Q.S. also acknowledges financial support from JSPS under Grant 18H01135.

[1] W. M. Pardridge, Drug transport across the blood-brain barrier, J. Cereb. Blood. Flow. Metab. 32, 1959 (2012). 
[2] J. Deseigne, C. Cottin-Bizonne, A. D. Stroock, L. Bocquet, and C. Ybert, How a "pinch of salt" can tune chaotic mixing of colloidal suspensions, Soft Matter 10, 4795 (2014).

[3] S. Xu, Z. Nie, M. Seo, P. Lewis, E. Kumacheva, H. A. Stone, P. Garstecki, D. B. Weibel, I. Gitlin, and G. M. Whitesides, Generation of monodisperse particles by using microfluidics: Control over size, shape, and composition, Angew. Chem. 44, 724 (2005).

[4] L. M. McDowell-Boyer, J. R. Hunt, and N. Sitar, Particle transport through porous media, Water Resour. Res. 22, 1901 (1986).

[5] R. Ashley, J.-L. Bertrand-Krajewski, and T. Hvitved-Jacobsen, Sewer solids-20 years of investigation, Water Sci. Technol. 52, 73 (2005).

[6] D. Vigolo, S. Radl, and H. A. Stone, Unexpected trapping of particles at a T junction, Proc. Natl. Acad. Sci. USA 111, 4770 (2014).

[7] K. K. Chen, C. W. Rowley, and H. A. Stone, Vortex dynamics in a pipe T-junction: Recirculation and sensitivity, Phys. Fluids 27, 034107 (2015).

[8] S. Shin, J. T. Ault, and H. A. Stone, Flow-driven rapid vesicle fusion via vortex trapping, Langmuir 31, 7178 (2015).

[9] J. T. Ault, A. Fani, K. K. Chen, S. Shin, F. Gallaire, and H. A. Stone, Vortex-Breakdown-Induced Particle Capture in Branching Junctions, Phys. Rev. Lett. 117, 084501 (2016).

[10] S. T. Chan, S. J. Haward, and A. Q. Shen, Microscopic investigation of vortex breakdown in a dividing T-junction flow, Phys. Rev. Fluids 3, 072201(R) (2018).

[11] D. Oettinger, J. T. Ault, H. A. Stone, and G. Haller, Invisible Anchors Trap Particles in Branching Junctions, Phys. Rev. Lett. 121, 054502 (2018).

[12] S. Duhr and D. Braun, Optothermal Molecule Trapping by Opposing Fluid Flow with Thermophoretic Drift, Phys. Rev. Lett. 97, 038103 (2006).

[13] B. Abécassis, C. Cottin-Bizonne, C. Ybert, A. Ajdari, and L. Bocquet, Boosting migration of large particles by solute contrasts, Nat. Mater. 7, 785 (2008).

[14] S. Shin, J. T. Ault, P. B. Warren, and H. A. Stone, Accumulation of Colloidal Particles in Flow Junctions Induced by Fluid Flow and Diffusiophoresis, Phys. Rev. X 7, 041038 (2017).

[15] S. M. Friedrich, J. M. Burke, K. J. Liu, C. F. Ivory, and T.-H. Wang, Molecular rheotaxis directs DNA migration and concentration against a pressure-driven flow, Nat. Commun. 8, 1213 (2017).

[16] J. T. Ault, S. Shin, and H. A. Stone, Diffusiophoresis in narrow channel flows, J. Fluid Mech. 854, 420 (2018).

[17] J. T. Ault, S. Shin, and H. A. Stone, Characterization of surface-solute interactions by diffusioosmosis, Soft Matter 15, 1582 (2019).

[18] M. Hermans, J. Gottmann, and F. Riedel, Selective, laser-induced etching of fused silica at high scanspeeds using KOH, J. Laser. Micro. Nanoen. 9, 126 (2014).

[19] N. Burshtein, S. T. Chan, K. Toda-Peters, A. Q. Shen, and S. J. Haward, 3D-printed glass microfluidics for fluid dynamics and rheology, Curr. Opin. Colloid Interface Sci. 43, 1 (2019).

[20] H. G. Weller, G. Tabor, H. Jasak, and C. Fureby, A tensorial approach to computational continuum mechanics using object-oriented techniques, Comput. Phys. 12, 620 (1998).

[21] D. S. Jang, R. Jetli, and S. Acharya, Comparison of the piso, simpler, and simplec algorithms for the treatment of the pressure-velocity coupling in steady flow problems, Numer. Heat Tr. A Appl. 10, 209 (1986).

[22] See Supplemental Material at http://link.aps.org/supplemental/10.1103/PhysRevFluids.5.024304 for Movies 1-4.

[23] B. V. Derjaguin, G. P. Sidorenkov, E. A. Zubashchenkov, and E. V. Kiseleva, Kinetic phenomena in boundary films of liquids, Kolloidn. Zh. 9, 335 (1947).

[24] B. V. Derjaguin, S. S. Dukhin, and A. A. Korotkova, Diffusiophoresis in electrolyte solutions and its role in mechanism of film formation from rubber latexes by method of ionic deposition, Kolloidn. Zh. 23, 53 (1961).

[25] J. L. Anderson and D. C. Prieve, Diffusiophoresis: Migration of colloidal particles in gradients of solute concentration, Sep. Purif. Methods 13, 67 (1984).

[26] J. L. Anderson, Colloid transport by interfacial forces, Ann. Rev. Fluid Mech. 21, 61 (1989). 
[27] D. C. Prieve, J. L. Anderson, J. P. Ebel, and M. E. Lowell, Motion of a particle generated by chemical gradients. Part 2. Electrolytes, J. Fluid Mech. 148, 247 (1984).

[28] A. Kar, T.-Y. Chiang, I. O. Rivera, A. Sen, and D. Velegol, Enhanced transport into and out of dead-end pores, ACS Nano 9, 746 (2015).

[29] S. Shin, E. Um, B. Sabass, J. T. Ault, M. Rahimi, P. B. Warren, and H. A. Stone, Size-dependent control of colloid transport via solute gradients in dead-end channels, Proc. Natl. Acad. Sci. USA 113, 257 (2016).

[30] S. Nishimura, H. Tateyama, K. Tsunematsu, and K. Jinnai, Zeta potential measurement of muscovite mica basal plane-aqueous solution interface by means of plane interface technique, J. Colloid Interface Sci. 152, 359 (1992).

[31] P. J. Scales, F. Grieser, T. W. Healy, L. R. White, and D. Y. Chan, Electrokinetics of the silica-solution interface: A flat plate streaming potential study, Langmuir 8, 965 (1992).

[32] I. Gusev and C. Horvath, Streaming potential in open and packed fused-silica capillaries, J. Chromatogr. A 948, 203 (2002).

[33] C. Schwer and E. Kenndler, Electrophoresis in fused-silica capillaries: The influence of organic solvents on the electroosmotic velocity and the zeta potential, Anal. Chem. 63, 1801 (1991).

[34] N. Cevheri and M. Yoda, Evanescent wave particle velocimetry measurements of zeta potentials in fused silica microchannels, Electrophoresis 34, 1950 (2013).

[35] S. Shin, J. T. Ault, J. Feng, P. B. Warren, and H. A. Stone, Low-cost zeta potentiometry using solute gradients, Adv. Mater. 29, 1701516 (2017).

[36] W. R. Dean, Note on the motion of fluid in a curved pipe, Philos. Mag. 4, 208 (1927).

[37] B. J. Kirby and E. F. Hasselbrink, Zeta potential of microfluidic substrates: 1. Theory, experimental techniques, and effects on separations, Electrophoresis 25, 187 (2004).

[38] T. Ishizaki, J. Hieda, M. A. Bratescu, N. Saito, and O. Takai, Biomimetic materials processing, Proc. SPIE 7404, 74040M (2009).

[39] B. Ball and D. W. Fuerstenau, A review of the measurement of streaming potentials, Miner. Sci. Eng. 5, 267 (1973).

[40] H. Ohshima and T. Kondo, Electrokinetic flow between two parallel plates with surface charge layers: electro-osmosis and streaming potential, J. Colloid Interface Sci. 135, 443 (1990).

[41] P. J. Sides and J. D. Hoggard, Measurement of the zeta potential of planar solid surfaces by means of a rotating disk, Langmuir 20, 11493 (2004).

[42] M. W. Shane, M. E. McCully, and M. J. Canny, Architecture of branch-root junctions in maize: Structure of the connecting xylem and the porosity of pit membranes, Ann. Bot. 85, 613 (2000).

[43] H. Meng, Z. Wang, Y. Hoi, L. Gao, E. Metaxa, D. D. Swartz, and J. Kolega, Complex hemodynamics at the apex of an arterial bifurcation induces vascular remodeling resembling cerebral aneurysm initiation, Stroke 38, 1924 (2007).

[44] A. P. Petroff, O. Devauchelle, H. Seybold, and D. H. Rothman, Bifurcation dynamics of natural drainage networks, Phil. Trans. Royal Soc. A 371, 20120365 (2013).

[45] Z. Chen, M. R. Bown, B. O'Sullivan, J. M. MacInnes, R. W. K. Allen, M. Mulder, M. Blom, and R. van't Oever, Performance analysis of a folding flow micromixer, Microfluid. Nanofluid. 6, 763 (2009).

[46] R. Milo and R. Phillips, Cell Biology by the Numbers (Garland Science, New York, NY, 2015).

[47] A. Hatch, A. E. Kamholz, K. R. Hawkins, M. S. Munson, E. A. Schilling, B. H. Weigl, and P. Yager, A rapid diffusion immunoassay in a T-sensor, Nat. Biotechnol. 19, 461 (2001).

[48] A. D. Stroock, S. K. Dertinger, A. Ajdari, I. Mezic, H. A. Stone, and G. M. Whitesides, Chaotic mixer for microchannels, Science 295, 647 (2002).

[49] T. J. Johnson, D. Ross, and L. E. Locascio, Rapid microfluidic mixing, Anal. Chem. 74, 45 (2002).

Correction: The previously published Figs. 4(e), 4(f), and 5(b) contained errors in the curve key and have been replaced. 